Article

\title{
Development of Singlet Oxygen Luminescence Kinetics during the Photodynamic Inactivation of Green Algae
}

\author{
Tobias Bornhütter ${ }^{1}$, Judith Pohl ${ }^{1}$, Christian Fischer ${ }^{1}$, Irena Saltsman ${ }^{2}$, Atif Mahammed ${ }^{2}$, \\ Zeev Gross ${ }^{2}$ and Beate Röder ${ }^{1, *}$ \\ 1 Department of Physics, Humboldt-Universität zu Berlin, Berlin 10099, Germany; \\ Tobias.Bornhuetter@physik.hu-berlin.de (T.B.); Judith.Pohl@physik.hu-berlin.de (J.P.); chrisf87@live.de (C.F.) \\ 2 Schulich Faculty of Chemistry, Technion-Israel Institute of Technology, Haifa 32000, Israel; \\ saltsman@tx.technion.ac.il (I.S.); chatif@tx.technion.ac.il (A.M.); chr10zg@tx.technion.ac.il (Z.G.) \\ * Correspondence: Beate.Roeder@physik.hu-berlin.de; Tel.: +49-30-2093-7625
}

Academic Editors: M. Graça P. M. S. Neves and M. Amparo F. Faustino

Received: 6 February 2016; Accepted: 7 April 2016; Published: 13 April 2016

\begin{abstract}
Recent studies show the feasibility of photodynamic inactivation of green algae as a vital step towards an effective photodynamic suppression of biofilms by using functionalized surfaces. The investigation of the intrinsic mechanisms of photodynamic inactivation in green algae represents the next step in order to determine optimization parameters. The observation of singlet oxygen luminescence kinetics proved to be a very effective approach towards understanding mechanisms on a cellular level. In this study, the first two-dimensional measurement of singlet oxygen kinetics in phototrophic microorganisms on surfaces during photodynamic inactivation is presented. We established a system of reproducible algae samples on surfaces, incubated with two different cationic, antimicrobial potent photosensitizers. Fluorescence microscopy images indicate that one photosensitizer localizes inside the green algae while the other accumulates along the outer algae cell wall. A newly developed setup allows for the measurement of singlet oxygen luminescence on the green algae sample surfaces over several days. The kinetics of the singlet oxygen luminescence of both photosensitizers show different developments and a distinct change over time, corresponding with the differences in their localization as well as their photosensitization potential. While the complexity of the signal reveals a challenge for the future, this study incontrovertibly marks a crucial, inevitable step in the investigation of photodynamic inactivation of biofilms: it shows the feasibility of using the singlet oxygen luminescence kinetics to investigate photodynamic effects on surfaces and thus opens a field for numerous investigations.
\end{abstract}

Keywords: singlet oxygen; photodynamic inactivation; phototoxicity; biofilms; corroles

\section{Introduction}

Biofilms play a major role in biofouling and the biodeterioration of construction materials. The biodeterioration of a building not only compromises its aesthetic appearance, but can also destroy cultural heritage when it comes to ancient buildings and even endanger people when the structural integrity is affected [1-4].

Established methods for the suppression or removal of biofilms have major drawbacks: mechanical removal of biofilms comes along with removal of construction material, while chemical approaches like the use of biocides pose environmental risks. This has motivated numerous efforts to find alternate ways to remove/reduce biofilms or supress their formation.

The functionalized surfaces approach appears to be a highly suitable and promising general tactic [5-8]. Most biofilms on outdoor surfaces contain phototrophic organisms, which need light to 
exist [9], and photocatalytic approaches have hence been adopted and displayed numerous interesting results up to now. The use of titanium dioxide in various forms stands out in that regard, though it was proven to be less effective for phototrophic organisms in field studies than under laboratory conditions. It was shown that titanium dioxide-functionalized surfaces can generate NOx species, due to electron transfer-based photocatalytic effects, and these species may act as fertilizers for the microorganisms [6-8,10-12].

Our aim is the development of functionalized surfaces, taking advantage of the photodynamic effect, as an attractive alternative to the photocatalytic effects that, e.g., titanium dioxide- functionalized surfaces, are based on. The idea of surface-immobilized photosensitizers for inhibiting the growth of biofilms is promising for several reasons. Efficient photosensitizers have been identified and developed with regard to their application in clinical photodynamic therapy (PDT) for many decades [13]. Even though the requirements for photosensitizers differ between PDT and the photodynamic inactivation (PDI) of microorganisms, a variety of photosensitizers for PDI are available [14]. They all have an intense UV/vis absorption spectrum, with quite a dominant absorption in the visible part in common. In contrast to titanium dioxide, which absorbs in the UV, surfaces functionalized with photosensitizers would use a much larger part of the Sun's emission spectrum reaching our planet. Another advantage of photosensitizers is their low to non-existing dark toxicity, having been developed for medical use in most cases. In contrast to that, the concern regarding the toxicity of titanium dioxide- and nanoparticle-based photocatalysis applications to people and environment is raising, as the use of titanium dioxide in several fields of application is increasing $[8,15,16]$.

Even though PDT is in widespread use today [13] and photodynamic inactivation of bacteria (PIB) is becoming an acknowledged approach [14,17-21], only a few examples on PDI of phototrophic organisms have been reported [22-25]. A first preliminary step towards developing photoactive surfaces using the photodynamic effect, was reported by Pohl et al., who revealed the feasibility of green algae inhibition using the photodynamic effect [25].

For advancing the PDI of phototrophic microorganisms beyond a mere proof of concept, we considered that the next step should be to evaluate the utilization of direct singlet oxygen $\left({ }^{1} \mathrm{O}_{2}\right)$ luminescence and photosensitizer fluorescence measurements to characterize and optimize the conditions for photodynamic inactivation of green algae. Fluorescence spectroscopy in general is a powerful tool; however, it was proven in the past that the combination of fluorescence spectroscopy and the time-resolved detection of the phosphorescence of generated ${ }^{1} \mathrm{O}_{2}$ is the most effective approach to investigate photodynamic inactivation processes. While the photosensitizer's fluorescence may be analysed for deducing on its location and possible aggregation, the analysis of the ${ }^{1} \mathrm{O}_{2}$ luminescence kinetics provides an insight on the microenvironment of the photosensitizer. This may be used for revealing the biochemical mechanism of the inactivation, thus allowing for optimization of the photosensitizer in terms of localisation and effective singlet oxygen generation [26,27].

\section{Results}

Figure 1 shows photographs, spatial fluorescence and ${ }^{1} \mathrm{O}_{2}$ phosphorescence plots of the algae reference, the $\mathrm{PCor}^{+}$-algae, and the TMPyP-algae samples after one day of incubation (on day 2). Photographs of the samples are presented in the first column, the spatial distribution of the fluorescence in the second and the spatial distribution of the ${ }^{1} \mathrm{O}_{2}$ phosphorescence in the third column. The photographs illustrate the sample geometry and the inhomogeneity due to the sample preparation technique. The fluorescence intensity in the plot equates the integral over the spectral range of 550-900 nm. The ${ }^{1} \mathrm{O}_{2}$ phosphorescence intensity shown in Figure 1 equates the fitted amplitude.

As expected, the algae reference is fluorescent, but devoid of any ${ }^{1} \mathrm{O}_{2}$ phosphorescence. The TMPyP-algae sample shows spatial correlation of fluorescence and ${ }^{1} \mathrm{O}_{2}$ phosphorescence even though the sharp edges of the sample that can be seen in the photograph and in the fluorescence plot appear smooth in the ${ }^{1} \mathrm{O}_{2}$ phosphorescence plot. In contrast to this high spatial correlation, there is 
hardly any spatial correlation between the photograph and the fluorescence plot on one hand and the ${ }^{1} \mathrm{O}_{2}$ phosphorescence plot on the other hand in the $\mathrm{PCor}^{+}$-algae sample.
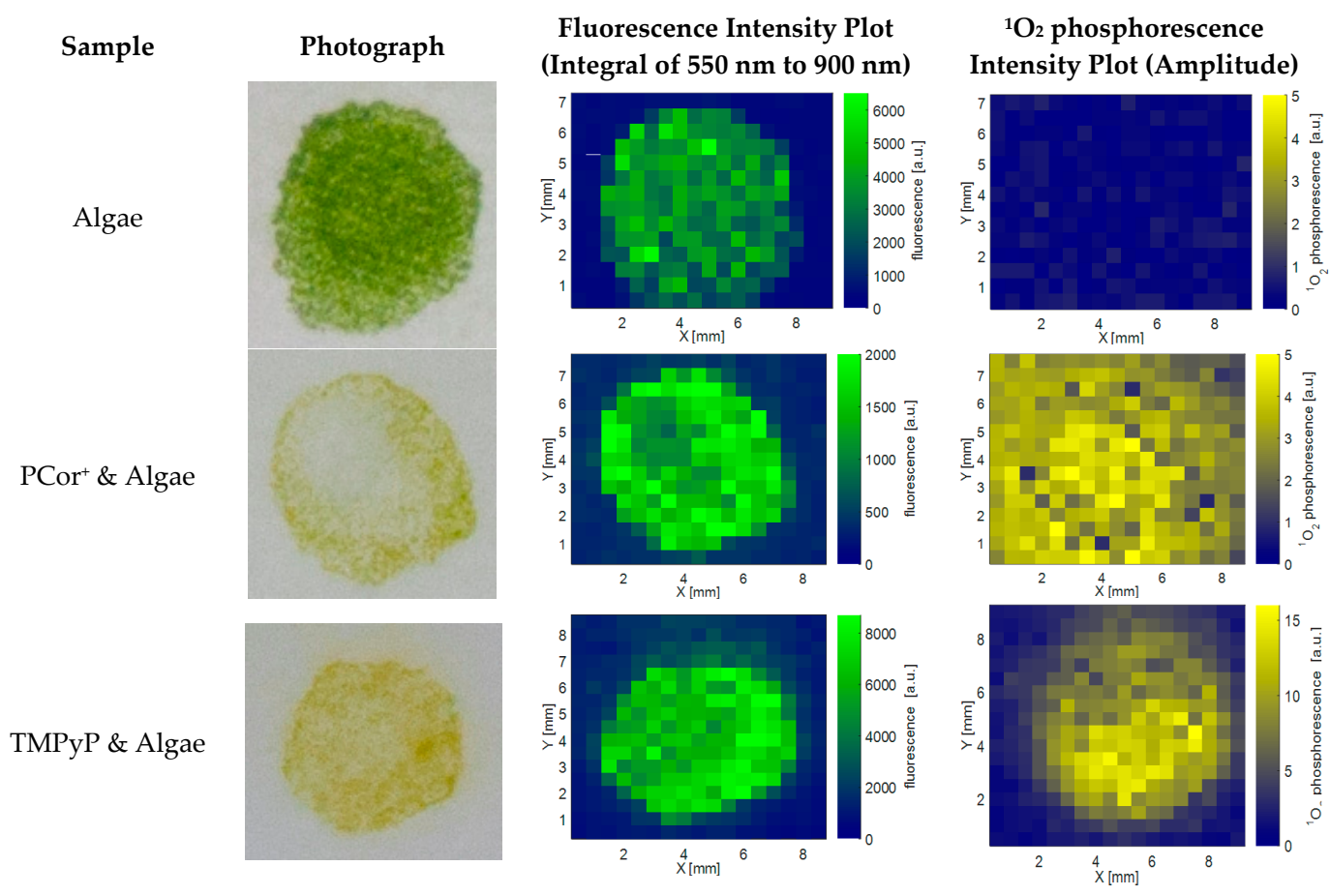

Figure 1. Comparison of the photographs, fluorescence intensity plots and singlet oxygen intensity plots of all samples. As expected, the algae reference does not display any ${ }^{1} \mathrm{O}_{2}$ phosphorescence. The TMPyP-algae-sample shows high spatial correlation of the photograph, and both the fluorescence and ${ }^{1} \mathrm{O}_{2}$ phosphorescence intensity plots. Interestingly, the ${ }^{1} \mathrm{O}_{2}$ phosphorescence intensity plot of the $\mathrm{PCor}^{+}$-algae-sample appears to contradict the fluorescence intensity plot and the photograph.

The abovementioned puzzling mismatch was resolved by recording the full fluorescence spectra of all samples on day 2. Figure 2 shows the fluorescence spectra summed up over five pixels of highest signal intensity, which reveals that: (a) the fluorescence intensity due to the algae's photosynthesis system is much stronger than that of the photosensitizers; (b) hardly any information can be drawn from the integrated fluorescence measurements of the algae/TMPyP combination because of the almost complete overlap of the emission spectra of the components; (c) the PCor ${ }^{+\prime}$ s fluorescence spectrum is located partly outside the algae emission, which may be used for its separate evaluation.
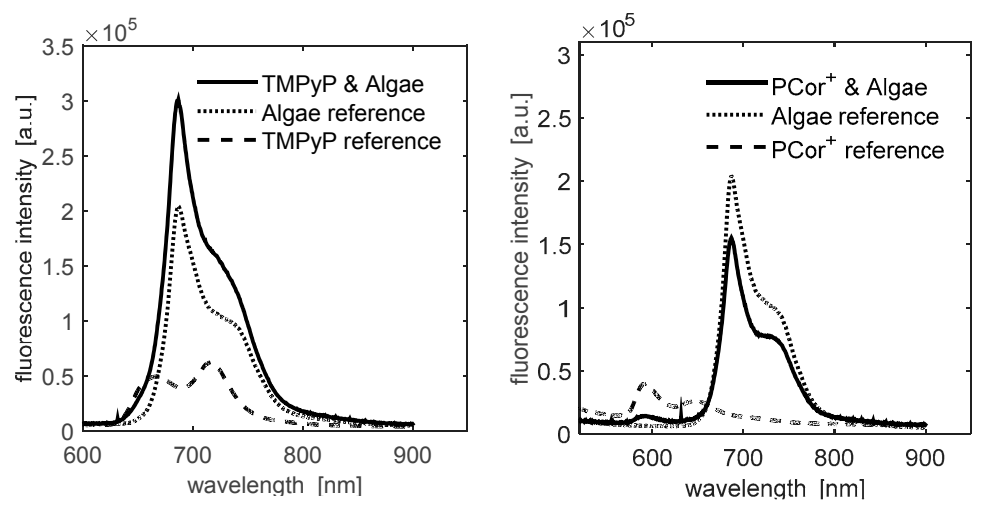

Figure 2. Comparison of fluorescence spectra of $\mathrm{PCor}^{+}$and TMPyP with algae to photosensitizer and algae reference (fluorescence intensity not corrected for different sample geometry). 
The latter hypothesis was confirmed by the results depicted in Figure 3. The left plot shows the fluorescence intensity as above, i.e., integrated from 550 to $900 \mathrm{~nm}$. The plot in the middle shows the fluorescence intensity integrated only over the range of $550-650 \mathrm{~nm}$, so as to isolate the $\mathrm{PCor}^{+/} \mathrm{s}$ fluorescence. This fluorescence plot is in correlation with the ${ }^{1} \mathrm{O}_{2}$ phosphorescence plot, indicating a diffusion of the photosensitizer onto the filter paper.
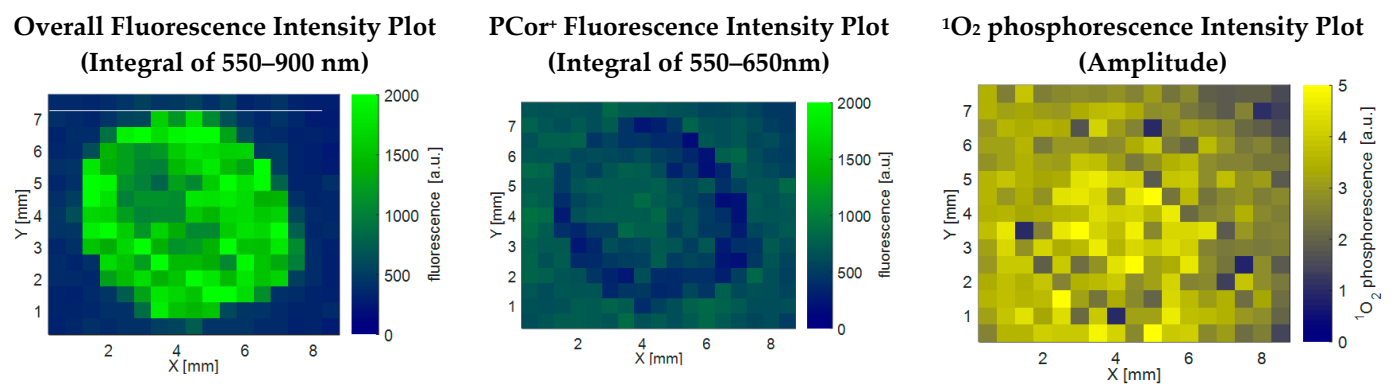

Figure 3. Comparison of the fluorescence intensity plots integrated over the whole recorded spectrum and the for the $\mathrm{PCor}^{+}$fluorescence relevant part of the recorded spectrum and the ${ }^{1} \mathrm{O}_{2}$ phosphorescence intensity plot for a $\mathrm{PCor}^{+}$-algae sample. In contrast to $\mathrm{TMPyP}, \mathrm{PCor}^{+}$appears to diffuse in the surrounding filter paper.

This evaluation of the spatial distribution of the fluorescence signal illustrates the importance of combining it with direct ${ }^{1} \mathrm{O}_{2}$ phosphorescence measurements. As mentioned in the Introduction, the ${ }^{1} \mathrm{O}_{2}$ kinetics provides information about the microenvironment of the photosensitizer. The most vital requirement for a detailed analysis of ${ }^{1} \mathrm{O}_{2}$ kinetics is a sufficient signal strength. To check this requirement and to explain how the later shown data was obtained, an excursion to the performed data analysis is necessary.

During this study, no changes were observed between the first and second scans of each sample on each day. Therefore, the signals of both scans per day were summed up pixel by pixel for each sample. The signal for the analysis and comparison of the samples and their temporal evolution were obtained by estimating the signal intensity of each pixel, evaluating the five pixels with highest intensity and calculating the sum of the signal of these five pixels.

To facilitate the comparison of the different signals, the standard biexponential model as described in [28] was extended by additional exponential terms in order to fit the data. The standard biexponential model for ${ }^{1} \mathrm{O}_{2}$ kinetics is the solution of a rate equation contemplating the generation and deactivation processes of ${ }^{1} \mathrm{O}_{2}$. The necessity of extending the biexponential model depends on the sample and is discussed along with the respective results. In general, the extension of the standard biexponential model was required because it describes ${ }^{1} \mathrm{O}_{2}$ kinetics in a homogeneous microenvironment, a condition that is apparently not experienced by the photosensitizers in the samples described in this study.

This approach was chosen to provide facile comparison of the signals and to visualize their development. As it is purely phenomenological for this study, no fitted parameter will be discussed here. The investigation of the applicability of this approach and the detailed analysis of the kinetics is an object of future work.

Figure 4 shows the raw data and the fitted model functions for the first two days, obtained in the aforementioned fashion. Even though the detailed analysis of the kinetics parameters is not the subject of this article, which presents a feasibility study, changes of the ${ }^{1} \mathrm{O}_{2}$ kinetics during the first two days of measurement and differences in the ${ }^{1} \mathrm{O}_{2}$ kinetics between the two photosensitizers as well as references and algae samples are clearly observed.

Figure 5 shows the ${ }^{1} \mathrm{O}_{2}$ kinetics of the $\mathrm{PCor}^{+}$reference sample and the $\mathrm{PCor}^{+}$-algae sample. The ${ }^{1} \mathrm{O}_{2}$ kinetics of the reference sample could be described by the unmodified biexponential model, i.e., without any extension. For the kinetics of the ${ }^{1} \mathrm{O}_{2}$ kinetics of the PCor ${ }^{+}$-algae sample, the biexponential model had to be extended by one additional simple exponential term. 


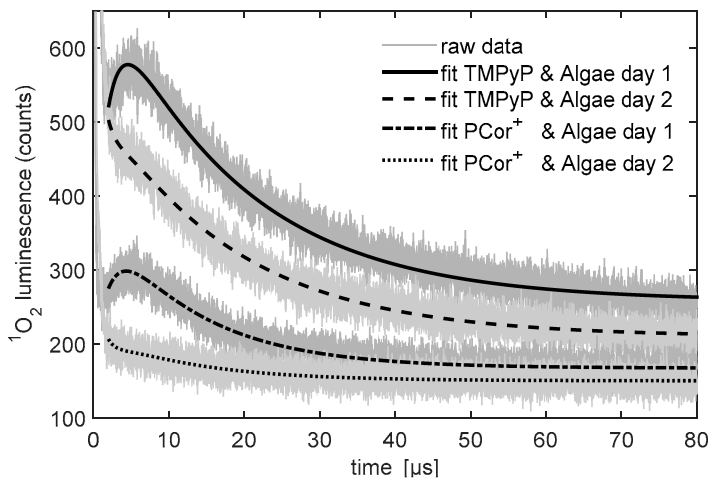

Figure 4. Raw data and fitted model functions.
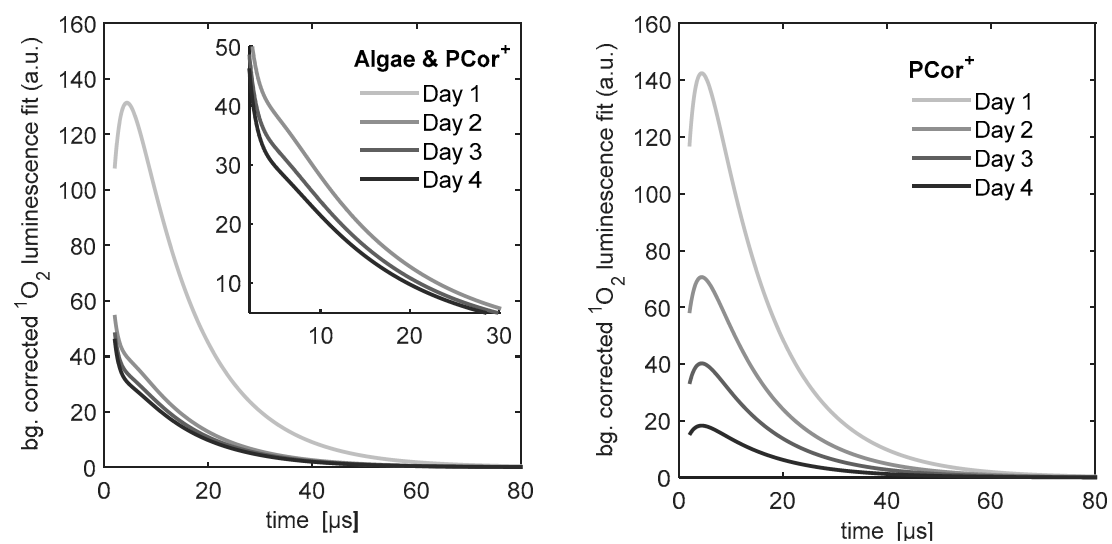

Figure 5. Temporal development of the singlet oxygen signals, measured on the surfaces of the PCor ${ }^{+}$ reference samples and the samples of $\mathrm{PCor}^{+}$incubated in green algae over four days.

Figure 6 shows the ${ }^{1} \mathrm{O}_{2}$ kinetics of the TMPyP reference sample and the TMPyP-algae sample. An additional simple exponential term has to be added to the biexponential model to describe the singlet oxygen luminescence kinetics, even for the TMPyP reference. The most complex kinetics were found for the TMPyP-algae sample, where two additional exponential terms had to be added to the biexponential model to fit the measured kinetic data. It must be pointed out that, as mentioned before, these fits were performed phenomenologically in order to facilitate the comparison of the data.
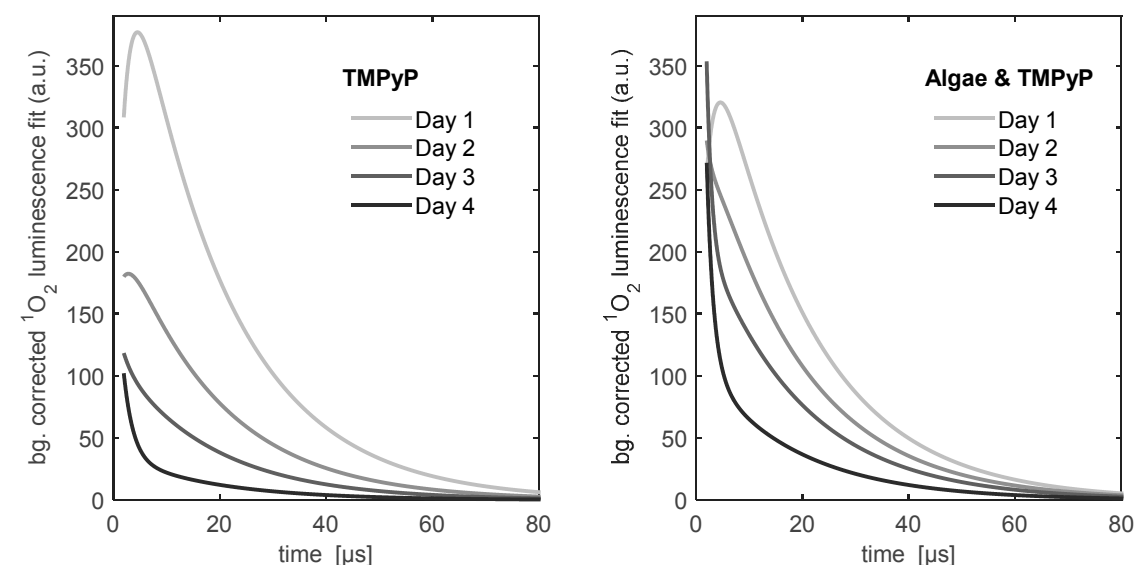

Figure 6. Temporal development of the singlet oxygen signals, measured on the surfaces of the TMPyP reference sample and the samples of TMPyP incubated in green algae over four days. 
Moreover, measurements at $1210 \mathrm{~nm}$ show a weak NIR signal for all TMPyP samples, in contrast to the algae references and all PCor $^{+}$samples where no signal was observed. The signal decreases exponentially over several $\mu \mathrm{s}$, corresponding to one of the additional exponential terms of the ${ }^{1} \mathrm{O}_{2}$ kinetics. Due to the kinetics and the design of both, the laser and the ${ }^{1} \mathrm{O}_{2}$ detection system, a measurement artefact is very unlikely. Since the signal is not significant in comparison to the here presented signals, it is not shown here.

Intra- vs. extra-cellular localization of the photosensitizers in the green algae after incubation was evaluated via CLSM and compared to the intrinsic fluorescence of chromophores (chlorophylls) inside the algae. In Figure 7, the resulting images of an untreated reference as well as samples incubated with either one of the two PS are shown alongside scattering images of the monitored cells. In contrast to scanning the fluorescence on macroscopic areas, CLSM imaging allows for separation of the fluorescence signals of PCor ${ }^{+}$and intrinsic chlorophylls by eliminating cross talk between channels using untreated reference samples. The (red coded) fluorescence of $\mathrm{PCor}^{+}$is apparent only at the periphery of the live cells (seen as circular lines of varying thickness), there is absolutely no overlap of signals attributed to intracellular chlorophyll and $\mathrm{PCor}^{+}$, and the few cases where $\mathrm{PCor}^{+\prime}$ s fluorescence appears round may be safely attributed to dead cells. The latter conclusion may also be deduced from the scattering image where no apparent cellular structures can be found in those regions.

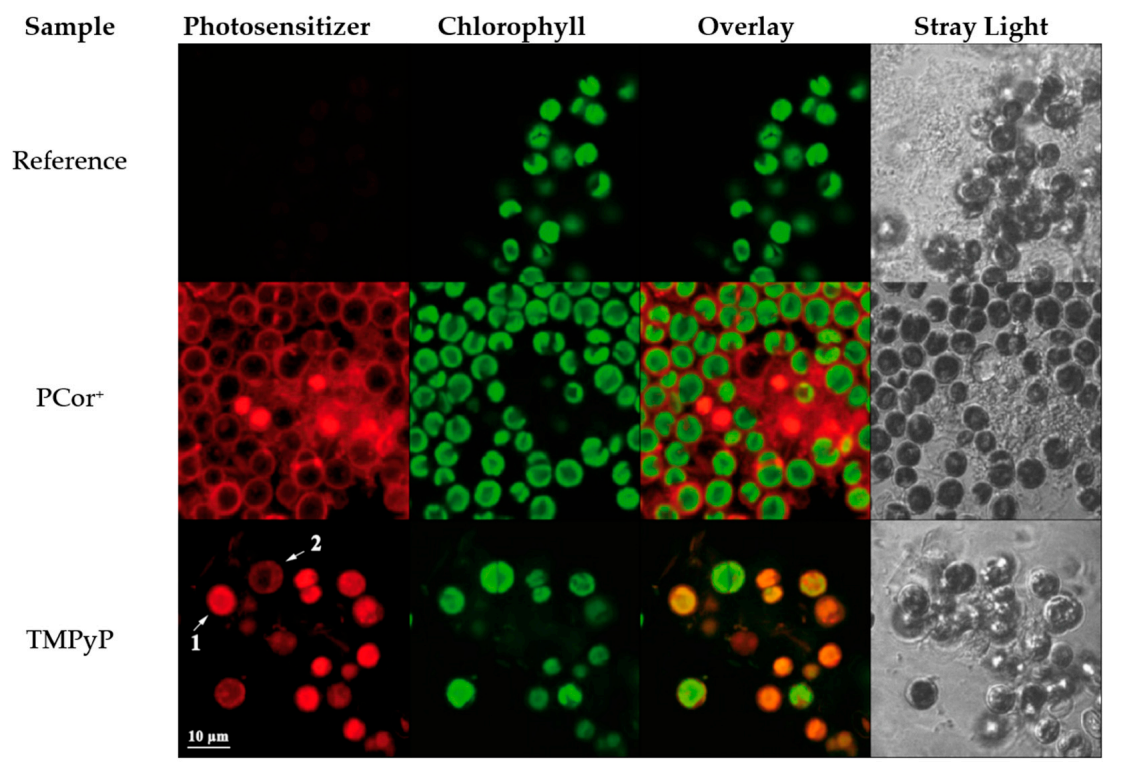

Figure 7. Localisation of the photosensitizer in Chlorella fusca var. vacuolata after three days of incubation. PCor ${ }^{+}$appears to locate outside the algae cells while TMPyP appears to accumulate inside the algae cells.

In contrast, fluorescence of TMPyP originates almost entirely from areas that also emit fluorescence of algae-chromophores. The distribution of the TMPyP fluorescence varies from fully filled circular areas (see Figure 7, arrow 1) to detailed structures (see Figure 7, arrow 2). The scattering image of the same sample shows cellular structures at all areas of fluorescence origin, with varying apparent viability of the cells.

\section{Discussion}

The obvious mismatch in the spatial correlation of fluorescence and ${ }^{1} \mathrm{O}_{2}$ phosphorescence for the PCor $^{+}$-algae sample in Figure 3 emphasizes once more the importance of direct ${ }^{1} \mathrm{O}_{2}$ phosphorescence detection for the investigation of photodynamic inactivation processes. The spatial distribution of the ${ }^{1} \mathrm{O}_{2}$ phosphorescence further indicates a diffusion of this photosensitizer into the surrounding filter paper, which is much more significant than for TMPyP. The ${ }^{1} \mathrm{O}_{2}$ phosphorescence examinations reveal 
a nearly homogeneous allocation for the $\mathrm{PCor}^{+}$-algae sample, while only the smoothing of the edges indicates a diffusion of TMPyP. This kind of effect would probably go unnoticed by looking only at the spatial correlation of the fluorescence, especially for TMPyP, where it is impossible to separate the TMPyP fluorescence and algae autofluorescence.

CLSM images clearly indicate a localisation of $\mathrm{PCor}^{+}$mainly onto the cell wall of the algae while TMPyP appears to accumulate inside the algae cell. With respect to future applications regarding the suppression of biofilms, photosensitizers accumulating and acting from the outside are clearly preferable.

The development of the ${ }^{1} \mathrm{O}_{2}$ phosphorescence kinetics of the PCor ${ }^{+}$reference shows a decrease of the signal with hardly any change in the kinetics. This indicates either a photobleaching of the photosensitizer or a change of the photosensitizer concentration on the investigated surface. Since $\mathrm{PCor}^{+}$is highly water soluble and did not show any signs of photobleaching in prior studies, the possibility of a diffusion of the photosensitizer in the BBM-agar film appears much more likely.

The development of the ${ }^{1} \mathrm{O}_{2}$ phosphorescence kinetics of the TMPyP reference raises more issues than that of the $\mathrm{PCor}^{+}$reference. The decrease of the signal indicates a similar diffusion in the BBM-agar film. In prior experiments, TMPyP showed such diffusion into the agar substrates.

The percentage of exponential increase in the signal, developing after 4 days, is due to unobvious effects that will have to be investigated in the future. The very weak luminescence at $1210 \mathrm{~nm}$, whose kinetics corresponds to one of the additional exponential terms of the ${ }^{1} \mathrm{O}_{2}$ kinetics, which was measured for all TMPyP samples, indicates an autophosphorescence of TMPyP. However, this auto-phosphorescence alone obviously cannot explain the signal development.

The development of the ${ }^{1} \mathrm{O}_{2}$ phosphorescence kinetics of all the algae-photosensitizer samples have two effects in common: after one day of incubation the ${ }^{1} \mathrm{O}_{2}$ phosphorescence kinetics changes drastically and an exponential increase in the signal is observed over time. Since the first measurement was performed right after the sample preparation, it may safely be assumed that nearly no interaction of algae and photosensitizer took place. That explains the similarity of the measurements of the photosensitizer reference measurements and the photosensitizer-algae samples on day one.

After one day of incubation, the ${ }^{1} \mathrm{O}_{2}$ phosphorescence kinetics of the PCor $^{+}$-algae sample reaches a nearly stable condition for the rest of the measurement period. Only the amplitude decreases slightly. A correlation of this decrease with cell viability is a reasonable assumption, which however must be further validated by viability assays. This behaviour is in line with the indications for localisation of the $\mathrm{PCor}^{+}$on the outside of the cell wall, since this kind of process should take place in a time period of less than a day. In this context, the development of the ${ }^{1} \mathrm{O}_{2}$ phosphorescence kinetics of the TMPyP-algae samples also correlates with the assumed localisation of the TMPyP inside the algae, as intracellular uptake of this and related porphyrins has been shown to require several days. In this study, no ${ }^{1} \mathrm{O}_{2}$ phosphorescence signal was measured on green algae reference samples, despite being formed during photosynthesis. This is due to the abundance of naturally occurring molecules like carotenoids inside phototrophic organisms, which act as quenchers for naturally generated amounts of ${ }^{1} \mathrm{O}_{2}$. As shown in [25], this does not hinder the photodynamic inactivation of green algae. It was shown herein for the first time that the measurement of ${ }^{1} \mathrm{O}_{2}$ phosphorescence kinetics on surfaces containing phototrophic organisms is possible. Even though the kinetics are complex, a distinct development of the kinetics over the four days of measurement that correlates with the localization of the photosensitizer can be observed.

\section{Materials and Methods}

An experimental setup was constructed for obtaining reproducible scans of luminescence on sample surfaces (Figure 8). It consists of an LDM-405D excitation laser (Omikron-Laserage, Rodgau-Dudenhofen, Germany) a cross table allowing one to scan a sample in the X and Y directions, a detection optics system coupling the excited luminescence into a fibre, movable in the $\mathrm{Z}$ direction, a TCMP-1270 Singlet Oxygen Luminescence Detection System by SHB Analytics (Berlin, Germany) and a C10083CAH Fluorescence Spectrometer by Hamamatsu (Hamamatsu, Shizuoka, Japan). 


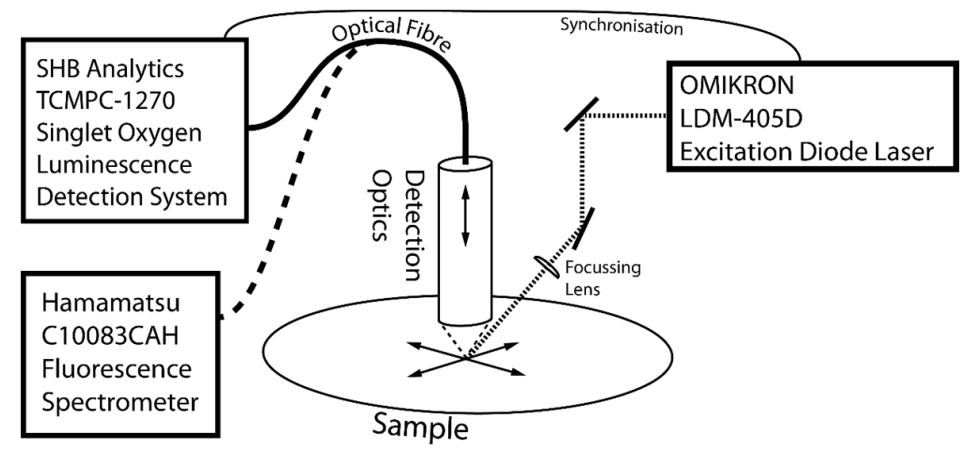

Figure 8. Scheme of ${ }^{1} \mathrm{O}_{2}$ scanning setup.

The TCMPC-1270 Singlet Oxygen Detection System allows for the time-resolved measurement of NIR-luminescence with highest sensitivity. Using a Hamamtsu H10330 photomultiplier tube, TCMPC electronics with time frames of $80 \mu \mathrm{s}, 160 \mu \mathrm{s}$ and $320 \mu \mathrm{s}$, and optical band path filters with centre wavelengths of $1270 \mathrm{~nm}$ and $1210 \mathrm{~nm}$ (both $\pm 15 \mathrm{~nm}$ ), it is optimized for the detection of very weak singlet oxygen luminescence signals. The Omikron LDM-405D laser is a $2 \mathrm{~W}$ diode laser of the TA Deepstar series with a wavelength of $405 \mathrm{~nm}$. With a modulation speed of up to $150 \mathrm{MHz}$ it is highly suitable as excitation laser in combination with the TCMPC-1270. The laser is PC controlled and modulated by the TCMPC-1270. The laser beam is focused to a spot of $c a .150 \mu \mathrm{m}$. The size of the laser spot is a good approximation for a lower boundary of the spatial resolution. The scanning motion is realized with custom build mechanics and software-controlled stepping motors.

The fluorescence detection can be realized by either coupling the detection fibre directly in the Hamamatsu C10083CAH fluorescence spectrometer or by using a dichroic mirror with a longpass cutoff wavelength of $1000 \mathrm{~nm}$ to detect fluorescence and ${ }^{1} \mathrm{O}_{2}$ phosphorescence simultaneously. To avoid the signal losses due to the dichroic mirror, in the later presented data, fluorescence and ${ }^{1} \mathrm{O}_{2}$ phosphorescence were measured sequentially.

A strain of green algae Chlorella fusca var. vacuolata (SAG 211-8b) was used as model organism for measurement of ${ }^{1} \mathrm{O}_{2}$ phosphorescence in phototrophic organisms. Suspension cultures of the algae were inoculated 3 days prior to experiments and grown at room temperature with shaking on a rotary shaker at $250 \mathrm{rpm}$ in Bold's Basal Medium (according to [29]). Illumination of the algal cultures during cultivation was realized with a daylight bulb (Photographic Lamp, $5400 \mathrm{~K}$, Realm Industrial GmbH, Berlin, Germany) in a day-night cycle of $12 \mathrm{~h}: 12 \mathrm{~h}$. All suspension cultures were provided with fresh medium directly before the start of any of the experiments.

$5,10,15,20$-Tetrakis(1-methylpyridinium-4-yl)porphyrin tetra( $p$-toluenesulfonate) (TMPyP) and 5,10,15-tris-(1-methylpyridinium-2-yl)corrolato-(trans-dihydroxo)phosphorus(V) $\left(\mathrm{PCor}^{+}\right)$, the corrole-based photosensitizer reported in $[17,25]$, were used as photosensitizers (Figure 9). TMPyP (CAS 36951-72-1) was purchased from Sigma-Aldrich Chemie GmbH (Munich, Germany), while the tricationic metallocorrole $\mathrm{PCor}^{+}$was synthesized according to [17].

For obtaining reproducible sample surfaces, algal cultures for the experiments were grown on filter paper $(\varnothing 90 \mathrm{~mm})$ on Bold's Basal Medium (BBM) agar plates. To this end, $200 \mu \mathrm{L}$ of algal suspension cultures with an initial cell density of $7 \times 10^{6} \mathrm{~mL}^{-1}$ were placed on a circular area with a diameter of 5-7 mm. Photosensitizers were added to the cultures prior to placement on the filter paper in a concentration of $5 \mu \mathrm{mol} / \mathrm{L}$. Samples of photosensitizer without green algae and green algae without photosensitizer were prepared as positive and negative controls for the singlet oxygen luminescence measurements, respectively.

The samples were covered with a fused silica window. This allows a high reproducibility of the singlet oxygen luminescence scan and prevents the samples from drying out. The samples were exposed to the $12 \mathrm{~h}$ day-night cycle using the $5400 \mathrm{~K} 20 \mathrm{~W}$ daylight bulbs for 4 days. 


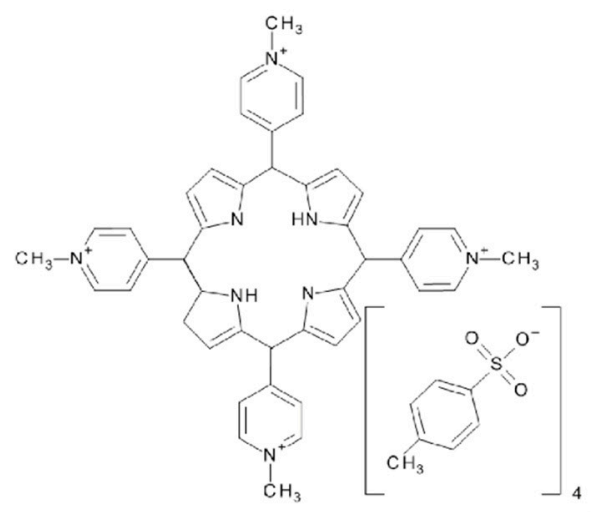

(a)

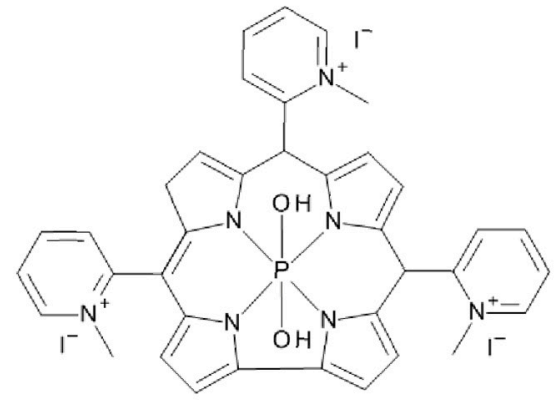

(b)

Figure 9. Chemical structures of the photosensitizers TMPyP and PCor ${ }^{+}$.

The singlet oxygen luminescence was scanned twice a day on an area of $8 \mathrm{~mm}$ by $6 \mathrm{~mm}$ with $1 \mathrm{~mm}$ pixel width in each direction. At each pixel the NIR luminescence signal was measured for $20 \mathrm{~s}$ using the $1270 \mathrm{~nm}$ bandwidth filter and an excitation energy of $0.5 \mu \mathrm{J}$. On days 1 and 4 , additional scans of the NIR luminescence at $1210 \mathrm{~nm}$ were performed under the same conditions.

To get information about the localisation of the photosensitizer in the green algae, incubated samples were examined by confocal laser scanning microscopy (CLSM) using a Fluo-ViewTM FV1000 (Olympus, Hamburg, Germany) after 3 days of incubation. Samples of Chlorella fusca var. vacuolata were grown on $2 \mathrm{~cm} \times 2 \mathrm{~cm}$ plastic slides for microscopy (Carl Roth GmbH, Karlsruhe, Germany) incubated in suspension cultures with initial cell density of $7 \times 10^{6} \mathrm{~mL}^{-1}$. The photosensitizers were applied to the cultures at a concentration of $5 \mu \mathrm{mol} / \mathrm{L}$. After 3 days of incubation, the plastic slides were rinsed with BBM and the remaining biofilms and precipitate was examined using a $405 \mathrm{~nm}$ laser for excitation. The fluorescence from the samples was detected on two channels: between 570 and $640 \mathrm{~nm}$ and above $640 \mathrm{~nm}$ using a bandpass filter. To avoid fluorescence crosstalk between those channels, excitation intensity and detector amplification were adjusted using untreated samples of the algae, until the intrinsic fluorescence signal is detected only in the second channel.

Acknowledgments: J.P. acknowledges Deutsche Bundesstiftung Umwelt (DBU) for funding. T.B. acknowledges DFG (RO 1042/33-1) for financial support. I.S., A.M. and Z.G. acknowledge the Israel Science Foundation for funding.

Author Contributions: Conception and execution of the experiments: Tobias Bornhütter, Judith Pohl, Christian Fischer and Beate Röder. Synthesis of photosensitizer: Irena Saltsman, Atif Mahammed, Zeev Gross. All authors have read and approved the final manuscript.

Conflicts of Interest: The authors declare no conflict of interest.

\section{Abbreviations}

The following abbreviations are used in this manuscript:

$\begin{array}{ll}\text { BBM } & \text { Bold's Basal Medium } \\ \text { PDT } & \text { Photodynamic Therapy } \\ \text { PDI } & \text { Photodynamic Inactivation } \\ \text { PIB } & \text { Photodynamic Inactivation of Bacteria } \\ { }^{1} \mathrm{O}_{2} & \text { Singlet Oxygen } \\ \text { TMPyP } & 5,10,15,20-\text { Tetrakis(1-methylpyridinium-4-yl)porphyrin tetra( } p \text {-toluenesulfonate) } \\ \text { PCor } & \text { 5,10,15-tris-(1-methylpyridinium-2-yl)corrolato-(trans-dihydroxo)phosphorus(V) } \\ \text { CLSM } & \text { Confocal Laser Scanning Spectroscopy }\end{array}$




\section{References}

1. Warscheid, T.; Braams, J. Biodeterioration of stone: A review. Int. Biodeterior. Biodegrad. 2000, 46, 343-368. [CrossRef]

2. Miller, A.Z.; Sanmartín, P.; Pereira-Pardo, L.; Dionísio, A.; Saiz-Jimenez, C.; Macedo, M.F.; Prieto, B. Bioreceptivity of building stones: A review. Sci. Total Environ. 2012, 426, 1-12. [CrossRef] [PubMed]

3. Turick, C.E.; Berry, C.J. Review of concrete biodeterioration in relation to nuclear waste. J. Environ. Radioact. 2016, 151, 12-21. [CrossRef] [PubMed]

4. Häubner, N.; Schumann, R.; Karsten, U. Aeroterrestrial microalgae growing in biofilms on facades-response to temperature and water stress. Microb. Ecol. 2006, 51, 285-293. [CrossRef] [PubMed]

5. Kochkodan, V.; Hilal, N. A comprehensive review on surface modified polymer membranes for biofouling mitigation. Desalination 2015, 356, 187-207. [CrossRef]

6. Graziani, L.; Quagliarini, E.; Osimani, A.; Aquilanti, L.; Clementi, F.; Yéprémian, C.; Lariccia, V.; Amoroso, S.; $\mathrm{D}^{\prime}$ Orazio, M. Evaluation of inhibitory effect of $\mathrm{TiO}_{2}$ nanocoatings against microalgal growth on clay brick façades under weak UV exposure conditions. Build. Environ. 2013, 64, 38-45. [CrossRef]

7. MacMullen, J.; Zhang, Z.; Dhakal, H.N.; Radulovic, J.; Karabela, A.; Tozzi, G.; Hannant, S.; Alshehri, M.A.; Buhé, V.; Herodotou, C.; et al. Silver nanoparticulate enhanced aqueous silane/siloxane exterior facade emulsions and their efficacy against algae and cyanobacteria biofouling. Int. Biodeterior. Biodegrad. 2014, 93, 54-62. [CrossRef]

8. Byrne, J.; Dunlop, P.; Hamilton, J.; Fernández-Ibáñez, P.; Polo-López, I.; Sharma, P.; Vennard, A. A Review of Heterogeneous Photocatalysis for Water and Surface Disinfection. Molecules 2015, 20, 5574-5615. [CrossRef] [PubMed]

9. Hallmann, C.; Rüdrich, J.; Enseleit, M.; Friedl, T.; Hoppert, M. Microbial diversity on a marble monument: A case study. Environ. Earth Sci. 2011, 63, 1701-1711. [CrossRef]

10. Wang, L.; Zhang, C.; Wu, F.; Deng, N. Photodegradation of aniline in aqueous suspensions of microalgae. J. Photochem. Photobiol. B Biol. 2007, 87, 49-57. [CrossRef] [PubMed]

11. Pacheco-Torgal, F.; Jalali, S. Nanotechnology: Advantages and drawbacks in the field of construction and building materials. Constr. Build. Mater. 2011, 25, 582-590. [CrossRef]

12. Gladis, F.; Schumann, R. Influence of material properties and photocatalysis on phototrophic growth in multi-year roof weathering. Int. Biodeterior. Biodegrad. 2011, 65, 36-44. [CrossRef]

13. Allison, R.R.; Downie, G.H.; Cuenca, R.; Hu, X.-H.; Childs, C.J.H.; Sibata, C.H. Photosensitizers in clinical PDT. Photodiagn. Photodyn. Ther. 2004, 1, 27-42. [CrossRef]

14. Preuss, A.; Zeugner, L.; Hackbarth, S.; Faustino, M.A.F.; Neves, M.G.P.M.S.; Cavaleiro, J.A.S.; Roeder, B. Photoinactivation of Escherichia coli (SURE2) without intracellular uptake of the photosensitizer. J. Appl. Microbiol. 2013, 114, 36-43. [CrossRef] [PubMed]

15. Bogdan, J.; Jackowska-Tracz, A.; Zarzyńska, J.; Pławińska-Czarnak, J. Chances and limitations of nanosized titanium dioxide practical application in view of its physicochemical properties. Nanoscale Res. Lett. 2015, 10. [CrossRef] [PubMed]

16. Shi, H.; Magaye, R.; Castranova, V.; Zhao, J. Titanium dioxide nanoparticles: A review of current toxicological data. Part. Fibre Toxicol. 2013, 10. [CrossRef] [PubMed]

17. Preuß, A.; Saltsman, I.; Mahammed, A.; Pfitzner, M.; Goldberg, I.; Gross, Z.; Röder, B. Photodynamic inactivation of mold fungi spores by newly developed charged corroles. J. Photochem. Photobiol. B Biol. 2014, 133, 39-46. [CrossRef] [PubMed]

18. Maisch, T.; Eichner, A.; Späth, A.; Gollmer, A.; König, B.; Regensburger, J.; Bäumler, W. Fast and effective photodynamic inactivation of multiresistant bacteria by cationic riboflavin derivatives. PLoS ONE 2014, 9, e111792. [CrossRef] [PubMed]

19. Eichner, A.; Gollmer, A.; Späth, A.; Bäumler, W.; Regensburger, J.; König, B.; Maisch, T. Fast and effective inactivation of Bacillus atrophaeus endospores using light-activated derivatives of vitamin B2. Photochem. Photobiol. Sci. 2015, 14, 387-396. [CrossRef] [PubMed]

20. Maisch, T. Resistance in antimicrobial photodynamic inactivation of bacteria. Photochem. Photobiol. Sci. 2015, 14, 1518-1526. [CrossRef] [PubMed] 
21. Maisch, T.; Baier, J.; Franz, B.; Maier, M.; Landthaler, M.; Szeimies, R.-M.; Bäumler, W. The role of singlet oxygen and oxygen concentration in photodynamic inactivation of bacteria. Proc. Nat. Acad. Sci. USA 2007, 104, 7223-7228. [CrossRef] [PubMed]

22. Drábková, M.; Marsálek, B.; Admiraal, W. Photodynamic therapy against cyanobacteria. Environ. Toxicol. 2007, 22, 112-115. [CrossRef] [PubMed]

23. Young, M.E.; Alakomi, H.-L.; Fortune, I.; Gorbushina, A.A.; Krumbein, W.E.; Maxwell, I.; McCullagh, C.; Robertson, P.; Saarela, M.; Valero, J.; et al. Development of a biocidal treatment regime to inhibit biological growths on cultural heritage: BIODAM. Environ. Geol. 2008, 56, 631-641. [CrossRef]

24. McCullagh, C.; Robertson, P.K.J. Photosensitized Destruction of Chlorella vulgaris by Methylene Blue or Nuclear Fast Red Combined with Hydrogen Peroxide under Visible Light Irradiation. Environ. Sci. Technol. 2006, 40, 2421-2425. [CrossRef] [PubMed]

25. Pohl, J.; Saltsman, I.; Mahammed, A.; Gross, Z.; Röder, B. Inhibition of green algae growth by corrole-based photosensitizers. J. Appl. Microbiol. 2015, 118, 305-312. [CrossRef] [PubMed]

26. Hackbarth, S.; Röder, B. Singlet oxygen luminescence kinetics in a heterogeneous environment-identification of the photosensitizer localization in small unilamellar vesicles. Photochem. Photobiol. Sci. 2015, 14, 329-334. [CrossRef] [PubMed]

27. Hackbarth, S.; Schlothauer, J.; Preuss, A.; Röder, B. Highly sensitive time resolved singlet oxygen luminescence detection using LEDs as the excitation source. Laser Phys. Lett. 2013, 10, 125702. [CrossRef]

28. Schlothauer, J.; Röder, B.; Hackbarth, S.; Lademann, J. In vivo detection of time-resolved singlet oxygen luminescence under PDT relevant conditions. SPIE Proc. 2010, 7551, 484-490.

29. Bischoff, H.W.; Bold, H.C. Some Soil Algae from Enchanted Rock and Related Algal Species; University of Texas: Austin, TX, USA, 1963.

Sample Availability: Samples of the compounds are available from the authors.

(C) 2016 by the authors; licensee MDPI, Basel, Switzerland. This article is an open access article distributed under the terms and conditions of the Creative Commons Attribution (CC-BY) license (http://creativecommons.org/licenses/by/4.0/). 